\title{
Daily Activities and Survival at Older Ages
}

Petra L. Klumb, $\mathrm{PhD}$

University of Fribourg, Fribourg, Switzerland

Heiner Maier, $\mathrm{PhD}$

Max Planck Institute for Demographic Research, Rostock, Germany

\begin{abstract}
Objective: This study tested the hypothesis that time spent on activities that are considered regenerative (e.g., resting), productive (e.g., housework), and consumptive (e.g., meeting friends) is associated with survival in persons aged 70 and older. Methods: An observational study with mortality follow-ups was carried out in the former West Berlin, Germany. The sample was stratified by age and sex, and it consisted of 473 persons aged 70 to 103 years. Study participants lived in the community as well as in institutions. Activity measures were assessed from 1990 to 1993 by structured interviews in the participants' homes. Cox regression was used to model survival from time of interview. The main outcome measure was survival on August 1, 2003. Results: Consumptive activities were related to survival (relative risk $=0.89,95 \%$ confidence interval 0.83 to 0.97 ), after several confounding factors were controlled for. The effect diminished over time. Discussion: Results support the idea that daily activities are linked to survival via a psychosocial pathway that might involve perceived quality of life. Consumptive activities (e.g., meeting friends, reading a novel) may contribute considerably to maintaining health and achieving longevity because they are performed on a daily basis and their effects may accumulate over the life course.
\end{abstract}

Keywords: daily activities, mortality, old age, production, Berlin Aging Study

$\mathrm{U}$ nderstanding the pattern in determinants of late-life survival becomes increasingly important as the population ages (Christensen \& Vaupel, 1996). It is well known that health-related behaviors such as smoking, drinking, and physical activity are related to mortality and survival. There are reports suggesting that other daily activities, such as meeting friends, shopping, and doing volunteer work, are associated with a longer life (Glass, de Leon, Marottoli, \& Berkman, 1999; Lennartsson \& Silverstein, 2001; Musick, 
Herzog, \& House, 1999). The etiologic mechanisms relating these daily activities to length of life are not well understood. Both physical and psychosocial pathways have been proposed. The physical component of activities such as hanging up laundry and cleaning the house exerts a direct influence on bodily processes. It improves musculoskeletal strength as well as cardiopulmonary performance, and it may reduce the risk of cardiovascular disease (Helmert, Herman, \& Shea, 1994). Physical activity also beneficially influences lipid and glucose metabolism as well as lipoprotein levels (Goldberg \& Elliot, 1985).

In addition to physical influences, psychosocial effects are thought to be in operation. Engagement in complex activities such as solving puzzles and reading novels affords learning and enjoyment (Baltes, 1996). Moreover, social activities such as chatting and playing board games may create a sense of belonging and emotional closeness. Involvement in this kind of activity has two consequences: a direct effect on well-being (Clark \& Watson, 1988) and a stress-buffering effect (House, Landis, \& Umberson, 1988).

Previous research on activities and survival has been hampered by the lack of a general theory that provides a well-defined conceptualization of activity types and their antecedents and consequences. Basing our study on Baltes's two-component model (Baltes, Maas, Wilms, Borchelt, \& Little, 1999), we distinguish two broad categories of daily activities (see Figure 1). On one hand, there are regenerative activities, which have to be carried out by physiological necessity (e.g., personal hygiene, eating, and resting). On the other hand, there are discretionary activities, which one can do by choice on the basis of individual abilities and preferences. The third-party criterion (Reid, 1934) serves to further subdivide discretionary activities-in this case, into productive and consumptive ones. If an activity is performed predominantly because of its outcomes and can therefore be delegated to a third party without losing its benefit (e.g., doing laundry, cleaning house, running errands), then it is productive. In industrialized societies, individuals of all ages delegate at least some productive activities to third parties-for example, when they purchase market products and services instead of producing them at

\footnotetext{
Authors' Note: We thank Hayden Bosworth, Karl Brehmer, Harvey Brenner, and Ursula Staudinger for their helpful comments. The present research was conducted in the context of the Berlin Aging Study (BASE), which is co-chaired by Paul B. Baltes and Karl Ulrich Mayer. BASE has been financially supported by several organizations, including the German Federal Ministry for Research and Technology (1989-1991, 13 TA 011 and 13 TA 011/A) and the German Federal Ministry for Family, Senior Citizens, Women, and Youth (1992-1998, 314-1722102/9 and 314-1722-102/9a). Please address correspondence to Petra L. Klumb, Department of Psychology, University of Fribourg, Rue de Faucigny 2, 1700 Fribourg, Switzerland; e-mail: petra.klumb@unifr.ch.
} 
Figure 1

A Theory-Based Categorization of All Activities Into Regenerative, Productive, and Consumptive Activities

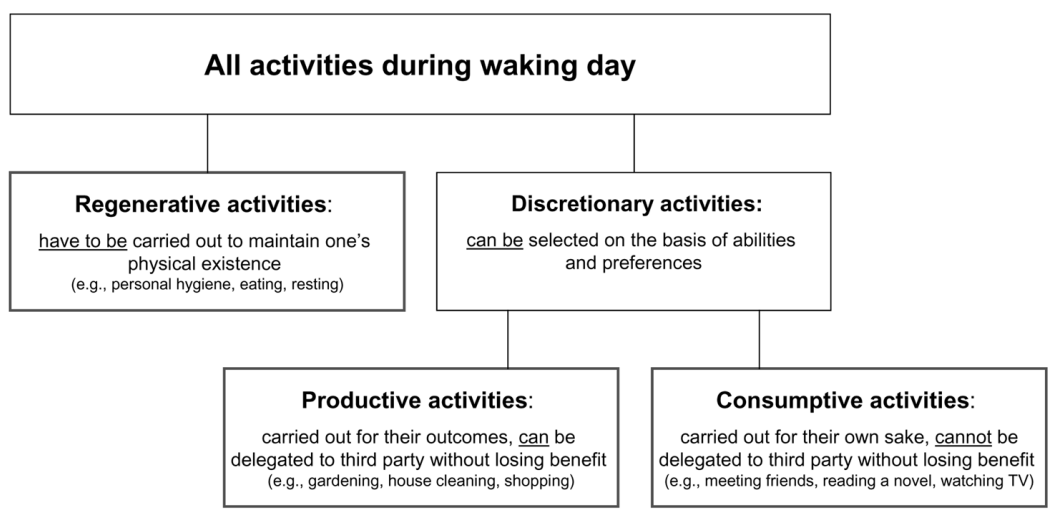

home. In contrast, if an activity is performed primarily for its own sake and cannot therefore be delegated to a third party without losing benefit (e.g., meeting friends, reading a novel, watching television), then it is consumptive.

According to this reasoning, two different types of benefits are connected with productive and consumptive activities. Benefits obtained from productive activities are mainly mediated through their outcomes. Psychosocial and health benefits accruing from productive activities can thus be maintained, to a large extent, by delegating them. Benefits accruing from consumptive activities, in contrast, are more direct and result from the process of performing the activity-in the microeconomic literature, they are called process benefits (Juster \& Stafford, 1991). Process benefits cannot be maintained by delegation of the activity to a third party. Consequently, we hypothesized that not engaging in consumptive activities has more damaging consequences for well-being, health, and longevity than not engaging in productive activities.

Some previous studies on the effects of activities adopted the assumption that health benefits increase monotonically with increasing participation in a particular activity. Alternatively, the microeconomic concept of decreasing returns on investment has been used to describe the relationship between daily activities and health outcomes (Bird \& Fremont, 1991). According to this 
concept, the relationship may level off beyond a certain point because additional time units allocated to an activity may not make any difference and may even be associated with decreasing benefits because of resource depletion. For instance, once a person has reached a state of relaxation, more time allocated to reading, playing games, or watching television may not improve this state any further. Thus, we hypothesized that a medium amount of time devoted to discretionary activities conveys the greatest survival benefit.

In sum, we examined two hypotheses. First, we expected that consumptive and, to a lesser extent, productive, but not regenerative, activities would be associated with survival. Second, we expected that benefits arising from consumptive and productive activities would be greatest for a medium duration of the activity, whereas a long or short duration would be associated with smaller benefits.

\section{Method}

\section{Participants and Sampling}

We used data from the first measurement occasion of the Berlin Aging Study (Baltes \& Mayer, 1999), which took place from 1990 to 1993. The study was designed to be representative of the West Berlin population aged 70 and older, while oversampling men and the very old. Samples originated from a random draw of addresses from the general registry (Landeseinwohneramt) of West Berlin. To obtain the final sample of 516 individuals stratified by age and sex, a much larger number of addresses had to be drawn. The study design consists of a hierarchical sequence of four levels of participation, with increasing numbers of variables but decreasing numbers of participants at each consecutive level: the verified parent sample $(n=1,908)$; the short-contact sample $(n=1,264)$; the intake assessment sample $(n=928)$; and the intensive protocol sample $(n=516)$, used in the present study, with its 14 sessions of multidisciplinary assessment. Extensive selectivity analyses (Lindenberger et al., 1999) showed the intensive protocol sample to be a somewhat positive selection of the parent sample. The magnitude of the selectivity effects was largest for general intelligence, but it did not exceed half a standard deviation for any of the analyzed domains of functioning. With one exception (dementia prevalence), selectivity effects did not interact with age or gender. Furthermore, comparisons of the sample with the Berlin census data showed no significant differences in indices such as marital status, proportion of institutionalized persons, and educational and income levels. 
The intensive protocol sample includes individuals ranging in age from 70 to 103 . The sample was stratified for age and sex, resulting in 43 women and 43 men in each of six age/cohort groups: 70-74 years (born 1915-1922), 75-79 years (born 1910-1917), 80-84 years (born 1905-1913), 85-89 years (born 1900-1908), 90-94 years (born 1896-1902), and 95-103 years (born 1883-1897). Given judgments made by one of us (P.K.), we excluded 31 participants from our analyses because of implausible activity data. Most of these persons were diagnosed as suffering from dementia.

Mortality status information and the date of death for the deceased participants were obtained from the State Registry Office. Mortality information on 12 individuals could not be obtained, because they had moved out of the Berlin area. These individuals were not considered in our analyses. This means that we utilized a total sample of 473 persons (230 women and 243 men) in our study.

\section{Measures}

Two types of measures were relevant: activity measures and a set of covariates. For the covariates, we rigorously chose common determinants of activity involvement and mortality (Rowe \& Kahn, 1998). The set of covariates included age, sex, years of education, measures of health and cognitive status, an indicator of whether participants lived with a spouse, and an indicator of whether they lived in an institution.

Activity measures. The Yesterday Interview (YI; Moss \& Lawton, 1982) was used to reconstruct the participants' day preceding the interview, from waking up to falling asleep. In a separate study, we compared self-reports assessed with the YI to time samples of activities in daily life, and we found acceptable agreement (Klumb \& Baltes, 1999b). The YIs took place in the participants' homes and lasted an average of about 50 min. Interview data were first categorized into 44 activity codes. We quantified the levels of intercoder agreement with the kappa statistic (Cohen, 1960). Kappas for all of the 44 activity codes were above .8 , suggesting high levels of intercoder agreement. The 44 activity codes were then condensed into 13 activity domains. Table 1 displays the average amount of time allocated to each of the 13 domains. Activity domains were then assigned to three broad activity categories: regenerative, productive, and consumptive (Klumb \& Baltes, 1999a).

Regenerative activities serve to maintain one's physical existence. This activity category comprised the activity domains resting and self-maintenance. 
Table 1

Average Time Allocated to Activity Categories and Domains $(N=473)$

\begin{tabular}{lcc}
\hline Category & $\begin{array}{c}\text { Duration in minutes } \\
\text { Domain }\end{array}$ & $\%$ of the waking day \\
& $M(S D)$ & $M(S D)$ \\
\hline Regeneration & $330(177)$ & $34.6(19.1)$ \\
$\quad$ Resting & $179(168)$ & $18.8(17.8)$ \\
Self-maintenance & $151(58)$ & $15.8(6.2)$ \\
Production & $150(130)$ & $15.5(13.1)$ \\
$\quad$ Gardening & $13(45)$ & $1.3(4.4)$ \\
Helping others & $3(18)$ & $0.3(1.9)$ \\
Housework & $107(98)$ & $11.0(10.1)$ \\
Paid work & $8(51)$ & $0.8(5.0)$ \\
Running errands & $21(40)$ & $2.1(4.1)$ \\
Consumption & $427(175)$ & $88.5(17.3)$ \\
$\quad$ Active leisure & $83(103)$ & $0.4(10.0)$ \\
Health-related activities & $9(21)$ & $4.5(5.1)$ \\
Locomotion & $44(57)$ & $6.6(8.8)$ \\
Social interaction & $63(81)$ & $9.7(10.0)$ \\
Reading & $93(96)$ & $18.4(13.8)$ \\
$\quad$ Watching television; listening & $179(135)$ & \\
$\quad$ to radio, records, tapes & &
\end{tabular}

For the distinction between productive and consumptive activities, we adopted the third-party criterion (Reid, 1934), which allows for a clear-cut categorization of activity domains. The category productive activities resulted from collapsing the following five activity domains: gardening, helping others (including volunteer work and provision of care for relatives), housework (including maintenance of home and possessions), paid work, and running errands. The six remaining domains were aggregated into the category consumptive activities. Specifically, this category comprised the following activity domains: active leisure (such as attending adult education courses or performing sports); health-related activities (including visits to doctors); locomotion (such as walking, driving with own vehicle, or riding as a passenger); reading; social interaction; and watching television or listening to radio, records, or tapes. Locomotion and health-related activities belong to this category because it is impossible to delegate them to a third person. Locomotion in the context of shopping and running errands, however, was coded as a productive activity. 
Education. We used the number of years spent in formal educational settings as an indicator of socioeconomic status. In addition to the number of years spent in elementary school and the different types of high schools in Germany (graduation after 10 to 13 years of schooling), this variable includes formal training both professional (e.g., apprenticeships) and academic (e.g., university). On average, participants in this sample had 10.8 years of education $(S D=2.3)$.

Number of diagnoses. We selected the number of moderate and severe diagnosed illnesses as an externally assessed indicator of participants' general health status. Diagnoses were determined in the course of consensus conferences of the research physician and psychiatrist (i.e., the physician and psychiatrist from the internal medicine unit and the psychiatry unit of the Berlin Aging Study, not the participants' personal doctors) and were based on a standardized summary of clinical findings from all diagnostic procedures. Illnesses diagnosed moderate and severe were summed up to form the variable number of diagnoses. On average, participants in this sample had eight diagnoses of moderate and severe illnesses $(S D=4)$.

Balance and gait. Functional health was represented by a unit-weighted composite of clinical assessments of balance and gait, the Romberg stance, and the turn-360 tasks (Tinetti, 1986). In the Romberg stance task, participants stood upright for about $1 \mathrm{~min}$, with legs as close together as possible, arms extended in front of the body, palms turned up, and eyes closed. Performance was scored by a physician on a 6-point scale ranging from no swaying to not able to stand upright at all. In the turn-360 task, participants were asked to perform a full turn around their body axes as fast as they could without risking a fall. The score corresponded to the number of steps needed to finish the circle. For the purpose of the present analyses, the balande and gait composite was represented as a $z$ score $(M=0, S D=1)$, with higher scores indicating higher levels of functional health.

Digit-Letter Test. We employed the Digit-Letter Test, a measure of perceptual speed, as an indicator of cognitive functioning. On a large table visible throughout the whole procedure, each of nine different letters was assigned to a digit. Participants were presented with tables containing six digits, and their task was to name the corresponding letters. The score consisted of the number of correct answers given within $3 \mathrm{~min}$. Stimulus presentation and data collection were supported by a Macintosh SE30 personal computer equipped 
with a Micro Touch Systems touch-sensitive screen. For the purpose of the present analyses, the Digit-Letter Test was represented as a $z$ score $(M=0$, $S D=1$, with higher scores reflecting higher levels of cognitive functioning.

Living with spouse. We included a dichotomous variable indicating whether a participant was living in the same household with his or her spouse or partner. This information was based on self-reporting by the participants and was verified by interviewer observation. In sum, 147 persons (31\%) lived with a spouse or partner in the same household; 326 persons (69\%) did not.

Living in institution. We included an indicator reflecting whether a participant was living in the community or in an institution. This information was based on self-reporting by the participants, and it was verified by interviewer observation. Overall, 409 persons (86\%) lived in the community; 64 persons (14\%) lived in institutions.

Vital status. The vital status of participants in the Berlin Aging Study is monitored at regular intervals. By August 2003 (representing a 10- to 13-year period after baseline assessment), 368 individuals ( $78 \%$ of this sample) were registered in the state records as deceased, and 95 persons (20\%) were registered as living. Ten individuals $(2 \%)$ were registered in the state records as alive in February 2000 but were subsequently lost as a result of follow-up issues. As is expected for a sample of this advanced age, a larger proportion of the oldest old had died (older than 85 years; $n=223$ decedents versus $n=7$ survivors) as compared to the younger age group (70-84 years; $n=145$ decedents versus $n=88$ survivors). As is also expected, a larger proportion of men had died ( $n=200$ decedents versus $n=37$ survivors) in comparison to women ( $n=168$ decedents versus $n=58$ survivors).

\section{Statistical Analyses}

Cox proportional hazards regression models (Cox, 1972) were evaluated for the effects of risk factors on survival from time of interview until August 1, 2003 (i.e., 10- to 13-year follow-up). We used the proportional hazards regression procedure (Allison, 1995) from the SAS software package to estimate Cox regression models. We report relative risks and their $95 \%$ confidence intervals. We used interaction terms (Activity Category $\times$ Time) to test the proportional hazards assumption. We used quadratic terms of the activity indicators to test the hypothesis of decreasing returns. 


\section{Figure 2}

Average Time Allocated to Regenerative, Productive, and

Consumptive Activities (in Minutes) for Women and Men Aged 70-84 and 85 and Older

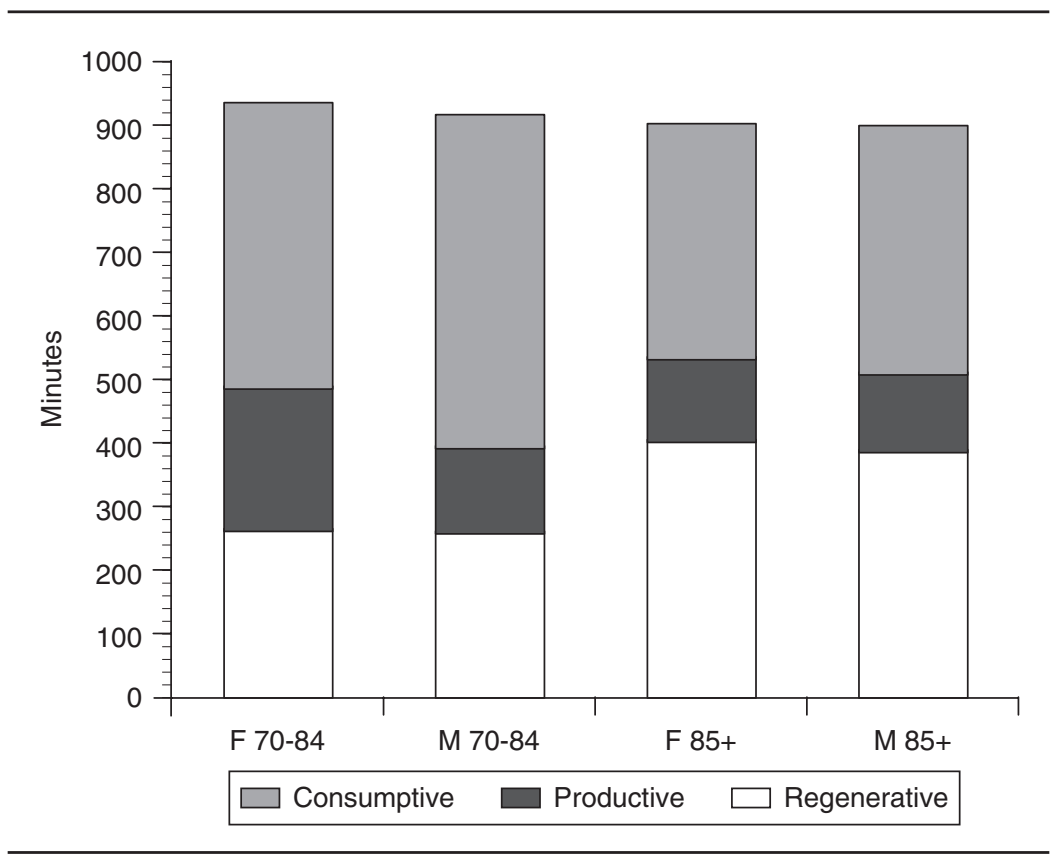

\section{Results}

Figure 2 displays the average levels of regenerative, productive, and consumptive activities by sex and age group (70-84 versus 85 and older). Average levels of regenerative activities for men and women aged 70-84 were both about $4 \mathrm{hr}$ and $20 \mathrm{~min}$ (less than a third of the waking day). For women and men aged 85 and older, the levels were about $6 \mathrm{hr}$ and $40 \mathrm{~min}$ (45\% of the waking day). Women aged 70-84 spent $3 \mathrm{hr}$ and $40 \mathrm{~min}$, on average, with productive activities (one fourth of their waking day), whereas the other groups allocated only $2 \mathrm{hr}$ to this kind of activity. The average time dedicated to consumptive activities varied between $8 \mathrm{hr}$ and $40 \mathrm{~min}$ (men aged 70-84) and $6 \mathrm{hr}$ (women aged 85 and older), with women aged 70-84 
(7 hr and $30 \mathrm{~min}$ ) and men aged 85 and older (6 hr and $30 \mathrm{~min})$ lying between these two extremes.

Because levels of participation in activities differed by age group and sex, we examined whether associations between activities and survival varied as a function of age and sex. We addressed this question by including interaction terms (Age $\times$ Activity Category, Sex $\times$ Activity Category) in the regression models. None of the interaction terms reached statistical significance (all $p>.05$ ) in the analyses adjusted for the set of covariates, suggesting that associations between activities and survival were similar across age and for women and men. Consequently, we omit age- and sex-specific analyses and report only results using data from the full sample $(N=473)$.

\section{Activities and Mortality Risk}

To test the association of everyday activities and mortality risk, we proceeded in two stages. We first determined the zero-order relationships, and in a second step, we evaluated mortality risks adjusted for the set of covariates (see Table 2).

In the unadjusted analyses, all activity types were significantly associated with risk of death. Higher levels of regenerative activities and lower levels of productive and consumptive activities were associated with an increased mortality risk. The magnitude of the associations was considerably reduced when we controlled for potential confounds and other activities. Regenerative and productive activities were no longer significantly associated with survival. However, after we controlled for the Activity $\times$ Time interaction, consumptive activities became associated with survival. The risk of death was then reduced by $11 \%$ for every additional hour spent on consumptive activities. The significant Activity $\times$ Time interaction suggests that the effect of consumptive activities decreased with time since baseline assessment.

\section{Decreasing Returns on Investment}

We examined the hypothesis of decreasing returns via curvilinear modeling of the activity categories. Specifically, we added quadratic terms of the amount of time spent on productive and consumptive activities to the models. After controlling for covariates, we found that none of the quadratic effects was statistically significant. This result is not consistent with the hypothesis that a medium amount of time devoted to an activity is associated with the most beneficial effect. 
Table 2

Mortality Risk Associated With Daily Activities and With Covariates $(N=473)$

\begin{tabular}{|c|c|c|}
\hline \multirow[b]{2}{*}{ Risk Factor } & \multicolumn{2}{|c|}{ Mortality Risk } \\
\hline & Unadjusted $^{\mathrm{a}}$ & Adjusted $^{\mathrm{b}}$ \\
\hline Regenerative (hours) & $1.17(1.14,1.21)^{*}$ & $1.00(0.95,1.07)$ \\
\hline Productive (hours) & $0.86(0.82,0.91)^{*}$ & $0.99(0.93,1.06)$ \\
\hline Consumptive (hours) & $0.90(0.87,0.94)^{*}$ & $0.89(0.83,0.97)^{*}$ \\
\hline Consumptive $\times$ Time & - & $1.02(1.01,1.03)^{*}$ \\
\hline Age (years) & $1.11(1.09,1.12)^{*}$ & $1.08(1.06,1.10)^{*}$ \\
\hline Sex $(0=$ female, $1=$ male $)$ & $1.33(1.08,1.63)^{*}$ & $1.62(1.26,2.08)^{*}$ \\
\hline Education (years) & $0.98(0.93,1.02)$ & $1.01(0.96,1.06)$ \\
\hline Diagnoses (number) & $1.08(1.06,1.11)^{*}$ & $1.07(1.04,1.10)^{*}$ \\
\hline Balance/gait $(z \text { score })^{\mathrm{c}}$ & $0.53(0.48,0.59) *$ & $0.81(0.71,0.93)^{*}$ \\
\hline Digit-Letter Test $(z \text { score })^{c}$ & $0.60(0.54,0.67)^{*}$ & $0.92(0.81,1.05)$ \\
\hline With spouse $(0=$ no, $1=$ yes $)$ & $0.80(0.64,1.00)^{*}$ & $1.10(0.83,1.45)$ \\
\hline In institution $(0=n o, 1=$ yes $)$ & $2.60(1.97,3.44)^{*}$ & $1.38(1.00,1.92)$ \\
\hline
\end{tabular}

Note: Relative risks are reported. Confidence intervals (95\%) for relative risks are shown in parentheses. The dash indicates that we did not examine the Consumptive $\times$ Time interaction without main effects.

a. Zero-order association of each risk factor with mortality.

b. Associations of risk factors with mortality after statistically controlling for all other factors.

c. Higher scores indicate higher levels of functioning.

$* p<.05 . * * p<.01$

\section{Discussion}

Time spent performing daily activities was related to mortality risk in this sample of persons aged 70 and older. In the unadjusted analyses, daily activity durations carried variance associated with common predictors of differential activity involvement and mortality risk. Above and beyond these confounding influences, consumptive activities were associated with a reduced risk of death. The effect weakened over time, as indicated by an Activity $\times$ Time interaction.

\section{Consumptive Activities Linked With Survival}

Consistent with our hypothesis was the finding that consumptive activities were related to survival. This result lends support to the proposal that there is a 
psychosocial pathway linking daily activities and survival. Consumptive activities affect perceived quality of life (Lawton, Moss, \& Fulcomer, 1986-1987) and afford opportunities for aspiring, experience of competence, and control. In contrast to some productive activities, such as household chores, consumptive activities are more challenging, and the demands that they place can be more easily adjusted to one's skill level or preferences because they are more freely chosen. They also yield more feedback and, on average, have more time devoted to them per day. Engagement in consumptive activities may thus affect mental and physical health and, ultimately, survival.

Our results suggest that the protective effect of consumptive activities diminishes over time. We speculate that this pattern is not caused by an actual weakening of the predictive strength but due to instability of the predictor during follow-up (Meinow, Kareholt, Parker, \& Thorslund, 2004)— that is, the decreased accuracy of the baseline data on consumptive activities. It is reasonable to assume that the proportion of persons whose time spent on consumptive activities changed after baseline increased during the follow-up period and, consequently, that the baseline information is less and less accurate as time passes.

\section{Productive Activities Not Linked With Survival}

In the adjusted analyses, time spent on productive activities was not related to longevity. It thus seems that decreasing time spent on productive activities (and increasing time spent on regenerative activities) is not directly linked to mortality but is rather a marker of physical and mental decline. The lack of an association between productive activities and long-term survival appears to be in conflict with previous reports positing that productive activities are linked with a longer life (Glass et al., 1999). Much of the earlier research investigating productive activities in relation to health outcomes focused on volunteering, that is, productive social activities (Luoh \& Herzog, 2002; Musick et al., 1999; Thoits \& Hewitt, 2001; Van Willigen, 2000). In contrast, the present study conceptualized productive activities more broadly, encompassing all activities that can be delegated without losing benefit. It was an empirical result of the present study that in this German sample the majority of productive activities were household chores-participation in paid and volunteer work was relatively low (cf. Table 1; Klumb \& Baltes, 1999a). Household chores differ from other productive activities, such as paid work and helping others (formally or informally). One difference is that-at least for people who live on their own - there are no others benefiting from the activity and, as Arendt (1981) emphasized, such an activity leaves no traces. Thus, household 
chores may often lack a characteristic of other productive activities, namely, confirming feedback and social recognition from others (Herzog, Kahn, Morgan, Jackson, \& Antonucci, 1989; Herzog \& Morgan, 1992).

The discrepant finding may also have resulted from the fact that other researchers (Glass et al., 1999) employed younger samples and represented activity involvement by frequency ratings instead of time use information. Frequency ratings are known to have two related disadvantages. First, it is possible to give high ratings for all cued activities because there is no frame of reference constraining the reports. In contrast, in a time budget, the relationships between durations of different activities tend to be negative, because engagement in one type of activity typically precludes simultaneous engagement in other activities. Second, frequency ratings are prone to third-variable confounding. Interindividual differences in personality may lead to differential activity reports, and these personality differences may be associated with health and longevity (see Chen \& Spector, 1991).

\section{Etiologic Mechanisms of the Psychosocial Pathway}

Our results suggest that consumptive, but not productive, activities are associated with survival. This very pattern of results indicates that psychosocial mechanisms are involved that operate independently of the physical pathway. Note that about $60 \%$ of the time spent on consumptive activities in this sample consisted of purely sedentary activities such as reading or watching television (see Table 1), whereas most productive activities required at least some physical exertion. If the effect were driven solely by low-intensity physical activity (Riddoch, 2000), then it should have been observed not only for consumptive activities but also for productive activities.

Which psychosocial processes can account for the link between daily activities and a longer life? It is likely that at least three different mechanisms are involved. First, it appears that the cognitive stimulation induced by cognitively challenging activities has beneficial effects for intellectual functioning (Schooler \& Mulatu, 2001) and reduces the risk of dementia (Wilson et al., 2002). Second, successful performance of chosen activities leads to the experience of competence and increases personal control. Both factors contribute to psychological well-being and alter the ways in which a person affectively and physiologically reacts to challenges (Karasek \& Theorell, 1990; Mirowsky \& Ross, 1998; Ross \& Drentea, 1998). Third, social interactions afford the experience of relatedness and emotional closeness, which are positively related to well-being (Reis, Sheldon, Gable, Roscoe, \& Ryan, 2000). Additionally, social affirmation and the knowledge that others benefit 
through one's activities increase the subjective meaning of an activity (Rowe \& Kahn, 1998; Thoits, 1983). Positive social interactions have been shown to alter basal neuroendocrine profiles and attenuate acute stress reactivity (Seeman, Berkman, Blazer \& Rowe, 1994; Seeman \& McEwen, 1996). Little is known yet about the relative contribution of these processes to the maintenance of health and longevity. But it is noteworthy that both experience of competence and social integration appear to exert their influence on health by promoting psychobiological recovery processes, and these recovery processes play a central role in the onset of age-related illnesses such as cardiovascular diseases, type 2 diabetes, and dementia (McEwen, 1998; Sapolsky, 1993). Recovery can take place not only during passive intervals of resting but also during active phases characterized by physical or mental activity (Karasek \& Theorell, 1990). For the beneficial effects to occur, moderately demanding consumptive activities such as crossword puzzling and playing cards with friends seem to be sufficient. We argue that, in terms of intervention, a reallocation of some of the time spent with resting to consumptive activities with low to moderate demands should be feasible, even for individuals with compromised health.

\section{Decreasing Returns on Investment}

We did not obtain empirical support for the idea that survival benefits do not increase monotonically with increasing involvement in activities. This finding diverges from previous studies on self-reported health in which the marginal returns on active and passive leisure were found to decrease steadily, eventually becoming negative (Bird \& Fremont, 1991; see also, Luoh \& Herzog, 2002; Musick et al., 1999). Because of the small size of the sample utilized in the present study, firm conclusions cannot be drawn here. Nevertheless, we believe that the issue of decreasing returns deserves further attention. Investigations into this area will benefit from the increased statistical power afforded by larger samples and from attention to the duration of single episodes.

\section{Strengths and Limitations}

A strong point of this study is that we employed a theory-guided activity categorization. Furthermore, we used a well-defined sample that was stratified by age and sex and included a considerable number of very old persons. Assessment of activity involvement based on the YI yielded reliable and valid activity information with a meaningful metric and the advantageous characteristics of time-budget data. Regarding covariates, we rigorously 
chose common determinants of activity involvement and mortality from the data protocol of the Berlin Aging Study in order to reduce the confounding effects of third variables. The chosen covariates were based not only on participants' self-reporting but also on performance tests and physician-observed diagnoses of illnesses. This selection of covariates minimized confounding through common method variance.

In addition to small sample size, at least three limitations should be kept in mind. First, only activity data from a single day was employed, and this day was not necessarily a typical one for all of the participants. It is thus likely that we underestimated the true size of the effects. Second, data did not contain sufficient information about the energy expenditure associated with performing an activity. Thus, it was not possible to rigorously control for variance in physical exertion, although this would have been desirable (Riddoch, 2000). Finally, causal effects of activities on survival cannot be inferred from this study, even though (a) activities preceded survival outcomes and (b) activity involvement was associated with mortality risk, after controlling for potential confounds. A risk factor can be called causal only if its manipulation changes the outcome (Kraemer et al., 1997), but we did not manipulate activity involvement in this study. However, the effects of altered engagement in consumptive activity can, in principle, be investigated in future intervention studies because time spent on consumptive activities is malleable and amenable to intervention. We suggest that consumptive activities may contribute considerably to the maintenance of health and to longevity because they are performed on a daily basis and their effects may accumulate over the life course.

\section{Conclusion}

Using time-budget data and a grouping of daily activities based on microeconomic theory, we found that consumptive activities were related to survival in persons aged 70 and older, although the protective effect diminished over time. This result supports psychological and sociological theorizing on the idea that daily activities and survival are linked through a psychosocial pathway, perhaps involving perceived quality of life, personal agency, and social recognition. Consumptive activities may prove to be a promising target for intervention because they are performed on a daily basis. Future research on activity engagement and survival may benefit from adopting the concept of decreasing returns on investment. Furthermore, researchers should aim at identifying the activity characteristics on which the effect on survival is based. 


\section{References}

Allison, P. D. (1995). Survival analysis using the SAS system: A practical guide. Cary, NC: SAS Institute.

Arendt, H. (1981). Vita activa oder Vom tätigen Leben [Vita activa or from active life]. München, Germany: Piper.

Baltes, M. M. (1996). Successful aging. In S. Ebrahim \& A. Kalache (Eds.), Epidemiology in old age (pp. 162-168). London: BMJ.

Baltes, M. M., Maas, I., Wilms, H.-U., Borchelt, M., \& Little, T. (1999). Everyday competence in old and very old age: Theoretical considerations and empirical findings. In P. B. Baltes \& K. U. Mayer (Eds.), The Berlin Aging Study: Aging from 70 to 100 (pp. 384-402). New York: Cambridge University Press.

Baltes, P. B., \& Mayer, K. U. (Eds.). (1999). The Berlin Aging Study: Aging from 70 to 100. New York: Cambridge University Press.

Bird, C. E., \& Fremont, A. M. (1991). Gender, time use, and health. Journal of Health and Social Behavior, 32, 114-129.

Chen, P. Y., \& Spector, P. E. (1991). Negative affectivity as the underlying cause of correlations between stressors and strains. Journal of Applied Psychology, 76, 398-407.

Christensen, K., \& Vaupel, J. W. (1996). Determinants of longevity: Genetic, environmental, and medical factors. Journal of Internal Medicine, 240, 333-341.

Clark, L. A., \& Watson, D. (1988). Mood and the mundane: Relations between daily life events and self-reported mood. Journal of Personality and Social Psychology, 54, 296-308.

Cohen, J. (1960). A coefficient of agreement for nominal scales. Educational and Psychological Measurement, 20, 37-46.

Cox, D. R. (1972). Regression models and life tables. Journal of the Royal Statistical Society: Series B: Statistical Methodology, 34, 187-220.

Glass, T. A., de Leon, C. M., Marottoli, R. A., \& Berkman, L. F. (1999). Population based study of social and productive activities as predictors of survival among elderly Americans. British Medical Journal, 319, 478-483.

Goldberg, L., \& Elliot, D. L. (1985). The effect of physical activity on lipid and lipoprotein levels. Medical Clinics of North America, 69, 41-55.

Helmert, U., Herman, B., \& Shea, S. (1994). Moderate and vigorous leisure-time physical activity and cardiovascular disease risk factors in West Germany, 1984-1991. International Journal of Epidemiology, 23, 285-292.

Herzog, A. R., Kahn, R. L., Morgan, J. N., Jackson, J. S., \& Antonucci, T. C. (1989). Age differences in productive activities. Journal of Gerontology: Social Sciences, 44, 129-138.

Herzog, A. R., \& Morgan, J. N. (1992). Age and gender differences in the value of productive activities. Research on Aging, 14, 169-198.

House, J. S., Landis, K. L., \& Umberson, D. (1988). Social relationships and health. Science, 241, 540-545.

Juster, F. T., \& Stafford, F. P. (1991). The allocation of time: Empirical findings, behavioral models, and problems of measurement. Journal of Economic Literature, 29, 471-522.

Karasek, R. A., \& Theorell, T. (1990). Healthy work. New York: Basic Books.

Klumb, P. L., \& Baltes, M. M. (1999a). Time use of old and very old Berliners: Productive and consumptive activities as functions of resources. Journal of Gerontology: Social Sciences, 54, S271-S278. 
Klumb, P. L., \& Baltes, M. M. (1999b). Validity of retrospective time-use reports in old age. Applied Cognitive Psychology, 13, 527-539.

Kraemer, H. C., Kazdin, A. E., Offord, D. R., Kessler, R. C., Jensen, P. S., \& Kupfer, D. J. (1997). Coming to terms with the terms of risk. Archives of General Psychiatry, 54, 337-343.

Lawton, M. P., Moss, M., \& Fulcomer, M. (1986-1987). Objective and subjective uses of time by older people. International Journal of Aging and Human Development, 24, 171-188.

Lennartsson, C., \& Silverstein, M. (2001). Does engagement with life enhance survival of elderly people in Sweden? The role of social and leisure activities. Journals of Gerontology: Social Sciences, 25, S335-S342.

Lindenberger, U., Gilberg, R., Little, T., Nuthmann, R., Pötter, U., \& Baltes, P. B. (1999). Sample selectivity and generalizability of results of the Berlin Aging Study. In P. B. Baltes \& K. U. Mayer (Eds.), The Berlin Aging Study: Aging from 70 to 100 (pp. 56-82). New York: Cambridge University Press.

Luoh, M. C., \& Herzog, A. R. (2002). Individual consequences of volunteer and paid work in old age: Health and mortality. Journal of Health and Social Behavior, 43, 490-509.

McEwen, B. S. (1998). Protective and damaging effects of stress mediators. New England Journal of Medicine, 338, 171-179.

Meinow, B., Kareholt, I., Parker, M. G., \& Thorslund, M. (2004). The effect of the duration of follow-up in mortality analysis: The temporal pattern of different predictors. Journal of Gerontology: Social Sciences, 59, S181-S189.

Mirowsky, J., \& Ross, C. E. (1998). Education, personal control, lifestyle and health-A human capital hypothesis. Research on Aging, 20, 415-449.

Moss, M. S., \& Lawton, M. P. (1982). Time budgets of older people: A window on four lifestyles. Journal of Gerontology, 37, 115-123.

Musick, M. A., Herzog, A. R., \& House, J. S. (1999). Volunteering and mortality among older adults: Findings from a national sample. Journal of Gerontology: Social Sciences, 54, S173-S180.

Reid, M. (1934). Economics of household production. New York: Wiley.

Reis, H. T., Sheldon, K. M., Gable, S. L., Roscoe, J., \& Ryan, R. M. (2000). Daily well-being: The role of autonomy, competence, and relatedness. Personality and Social Psychology Bulletin, 26, 419-435.

Riddoch, C. (2000). Social and productive activities in elderly people-Activities have been confused [Letter]. British Medical Journal, 320, 184.

Ross, C. E., \& Drentea, P. (1998). Consequences of retirement activities for distress and sense of personal control. Journal of Health and Social Behavior, 39, 317-334.

Rowe, J. W., \& Kahn, R. L. (1998). Successful aging. New York: Pantheon Books.

Sapolsky, R. M. (1993). Potential behavioral modification of glucocorticoid damage to the hippocampus. Behavioral Brain Research, 57, 175-182.

Schooler, C., \& Mulatu, M. S. (2001). The reciprocal effects of leisure time activities and intellectual functioning in older people: A longitudinal analysis. Psychology and Aging, 16, 466-482.

Seeman, T. E., Berkman, L. F., Blazer, D., \& Rowe, J. (1994). Social ties and support and neuroendocrine function: MacArthur studies of successful aging. Annals of Behavioral Medicine, $16,95-106$.

Seeman, T. E., \& McEwen, B. S. (1996). Impact of social environment characteristics on neuroendocrine regulation. Psychosomatic Medicine, 58, 459-471. 
18 To be published in : Journal of Aging and Health, vol 19, 4 ; pp. 594-611

Thoits, P. A. (1983). Multiple identities and psychological well-being: A reformulation and test of the social isolation hypothesis. American Sociological Review, 48, 174-187.

Thoits, P. A., \& Hewitt, L. N. (2001). Volunteer work and well-being. Journal of Health and Social Behavior, 42, 115-131.

Tinetti, M. E. (1986). A performance-orientated assessment of mobility problems in elderly patients. Journal of the American Geriatrics Society, 34, 119-126.

Van Willigen, M. (2000). Differential benefits of volunteering across the life course. Journal of Gerontology: Social Sciences, 55, S308-S318.

Wilson, R. S., de Leon, C. F. M., Barnes, L. L., Schneider, J. A., Bienias, J. L., Evans, D. A., et al. (2002). Participation in cognitively stimulating activities and risk of incident Alzheimer disease. Journal of the American Medical Association, 287, 742-748. 